\title{
Design of an Axial Transonic Rotor by Modified Spanwise Loading Distribution
}

\author{
A. Shahsavari ${ }^{1}$, M. Nili-Ahmadabadi ${ }^{1 \dagger}$, E. Shirani ${ }^{1}$ and K. Chun $\mathrm{Kim}^{2}$ \\ ${ }^{1}$ Department of Mechanical Engineering, Isfahan University of Technology, Isfahan 8415683111, Iran \\ ${ }^{2}$ Pusan National University, Busan 609-735, South Korea
}

†Corresponding Author Email: m.nili@iut.ac.ir

(Received March 3, 2020; accepted June 25, 2020)

\begin{abstract}
Improving the aerodynamic performance of the transonic fan in a turbofan engine can be beneficial for both the fuel consumption and maneuverability of an airplane. Deep insight into the supersonic aerodynamics is needed for the simultaneous improvement of all operating parameters of a transonic rotor, including the pressure ratio, efficiency, and surge margin. A design method was developed for an axial transonic rotor by using a combination of radial equilibrium theory, the free vortex method, and a distributed span-wise diffusion factor. The loading distribution obtained by this method was the highest in the hub section and gradually decreased in the tip section. To evaluate the method, a transonic rotor was designed using the geometric parameters and operating conditions of NASA rotor 67. A code was developed to determine a geometry for the new rotor and to modify it. The code was coupled with a RANS flow solver for 3D modification of the new designed rotor. Only standard multi- and double-circular arc airfoils were applied in different radial sections of the new rotor with no blade profile optimization. The results of the RANS equations solution for the new designed rotor showed $1.5 \%$ higher efficiency, $3 \%$ higher pressure ratio, and more than 1.5 times larger operating range in comparison to NASA rotor 67 . The new designed method seems to be an efficient approach that not only improved the efficiency and pressure ratio but also increased the operating range of an axial transonic rotor.
\end{abstract}

Keywords: Axial Fan; De-Haller number; Numerical simulation; Radial equilibrium; Rotor design procedure.

\section{NOMENCLATURE}

\begin{tabular}{|c|c|c|c|}
\hline AR & chord & $\mathrm{T}$ & temperature \\
\hline $\mathrm{Cd}$ & chord length & $\mathrm{t}$ & blade maximum thickness \\
\hline DCA & double circular arc & TE & trailing edge \\
\hline DF & diffusion factor & $\mathrm{U}$ & blade linear speed \\
\hline $\mathrm{dH}$ & de Haller number & $\mathrm{V}$ & absolute velocity \\
\hline f & target computational fucntion & $\mathrm{W}$ & relative velocity \\
\hline HTR & hub-to-tip-ratio & $\mathrm{z}$ & axial component of coordinate \\
\hline $\mathrm{h}$ & enthalpy & & \\
\hline $\mathrm{i}$ & incidence angle & $\alpha$ & flow angle \\
\hline $\mathrm{k}$ & grid level & $\beta$ & blade angle \\
\hline$l$ & relative grid size & $\delta$ & deviation angle \\
\hline $\mathrm{LE}$ & leading edge & $\varepsilon$ & error \\
\hline $\mathrm{m} \cdot$ & mass flow rate & $\eta$ & efficiency \\
\hline MCA & multi circular arc & $\theta$ & circumferential \\
\hline $\mathrm{N}$ & number of elements & & coordinate \\
\hline $\mathbf{O}$ & order of error & $\lambda$ & blade stagger angle \\
\hline $\mathrm{p}$ & pressure & $\mu$ & fluid viscosity \\
\hline r & radius or radial direction & $\rho$ & fluid density \\
\hline RANS & Reynolds Averaged Navier Stokes & $\bar{\tau}$ & shear and eddy stress tensor \\
\hline re & blade leading and trailing edge radius & $\varphi$ & camber angle \\
\hline $\mathrm{Rn}$ & reaction & $\psi$ & loading coefficient \\
\hline $\mathrm{r}_{\mathrm{p}}$ & total pressure ratio & $\Omega$ & rotor rotational speed \\
\hline$S M$ & surge margin & $\Omega_{\mathrm{N}}$ & nominal rotational speed \\
\hline
\end{tabular}


Subscripts

0 total or stagnation properties

1 blade inlet

2 blade outlet

$\mathrm{r} \quad$ radial direction t total

ss supersonic

sb subsonic

$\mathrm{z} \quad$ axial

$\theta \quad$ circumferential

\section{INTRODUCTION}

Due to their high propulsive efficiency, turbofan engines are currently the most popular propulsion system for different civil or military aircrafts (Benzakein 2014; Choi et al. 2020). The transonic rotor of a fan is one of the main components of a turbofan engine. The fan efficiency, operating range, and pressure ratio play important roles in the efficiency of a turbofan engine. Even a small increase in the efficiency and pressure ratio of the fan can be beneficial for the fuel consumption of an engine. Additionally, improving the operating range of the fan is necessary for the maneuverability of the airplane.

Enhancing all of these parameters simultaneously requires a deep insight into the aerodynamic design of axial transonic rotor. There are many studies on using optimization techniques to improve the performance of axial transonic rotors (Luo et al. 2014; Oyama et al. 2004; Samad et al. 2008; Walther \& Nadarajah 2013; Yang et al. 2018). Optimization has been applied to the blade profile (Tang et al. 2017), stacking line (Gao et al. 2017; Sun et al. 2019), and tip clearance (Danish et al. 2016; Ren \& Gu 2016), which are the most important geometric parameters. Recent studies on turbomachinery have mostly concentrated on the optimization of a specific geometry rather than developing a new design. Optimizing the geometry can be beneficial for the performance of turbomachinery while the main goal of an optimization is restricted by many constraints.

Many studies investigated the design parameters such as loading, reaction, and velocity triangles (Dickens \& Day 2011). Free vortex method is a conventional design procedure that has been used for many years by many designers to design axial compressors (Saravanamuttoo et al. 2001). This method has many advantages that make it popular for transonic rotors. Free vortex blades with more camber on the hub section (for subsonic flow) and less blade camber on the tip section (for supersonic flow) have better aerodynamic performance and more structural strength. But despite all these advantages, the free vortex method provides just a preliminary design that can be modified for enhanced rotor performance. Recently, a non-free design method was developed using constant diffusion to redesign an axial transonic compressor (Shahsavari \& Nili-Ahmadabadi 2017). The method was based on a constant diffusion along the whole span and was upgraded to be used in axial transonic fan with a higher tip Mach number (Shahsavari \& Nili-Ahmadabad 2016). However, despite the higher pressure ratio and bypass air, the designed fan with a constant diffusion factor had a weak aerodynamic efficiency. In the present study, a new design method was developed by combining the free vortex method and a distribution of variable span-wise diffusion factor to improve the efficiency, pressure ratio, and operating range of an axial flow rotor. A 3D compressible and viscous flow solver was used to investigate the performance of the transonic rotor designed by the new design procedure.

\section{DESIGN PROCEDURE}

The new design methodology combines the radial equilibrium theory for turbomachinery with a constant or variable span-wise diffusion factor to redesign a rotor. The radial equilibrium theory at the outlet of a rotor is represented in Eq. (1):

$$
\frac{\partial h_{0}}{\partial r}=v_{z} \frac{\partial v_{z}}{\partial r}+\frac{v_{\theta}}{r} \frac{\partial\left(r v_{\theta}\right)}{\partial r}
$$

The radial component of the velocity and radial variation of the entropy ( $\mathrm{Wu} \&$ Wolfenstein 1950) are neglected in this equation.

According to the previous non-free vortex design methodology (Shahsavari \& Nili-Ahmadabad 2016), a constant diffusion factor was beneficial for axial rotors near the hub section. Consequently, a constant diffusion can still be implemented in a blade design at $20 \%$ of the span. This study employs a variable diffusion factor in the radial equilibrium theory for the higher-span sections. The main purpose of implementing a variable diffusion factor distribution is to prevent high losses, especially in the tip section of the blades with higher Mach number. The diffusion is represented by the de Haller number as the ratio of the outlet to inlet relative velocity. The axial component of the outlet velocity for a specific de Haller number can be calculated according to Eq. (2):

$v_{z 2}=\sqrt{\left(d H \cdot w_{1}\right)^{2}-\left(U-v_{\theta 2}\right)^{2}}$

The derivative of Eq. (2) is used to calculate the first term on the right hand side of Eq. (1). The de Haller number is described as a function of the radius in Eq. (3). The number starts to decrease gradually towards the tip section with a switch function.

$$
\begin{aligned}
& v_{z 2} \frac{\partial v_{z 2}}{\partial r}=d H \cdot \frac{\partial(d H)}{\partial r} \cdot\left[v_{z 1}{ }^{2}+(r . \Omega)^{2}\right]+ \\
& d H^{2} \cdot\left(r \cdot \Omega^{2}\right)-\left(r \cdot \Omega-v_{\theta 2}\right)\left(\Omega-\frac{\partial v_{\theta 2}}{\partial r}\right)
\end{aligned}
$$




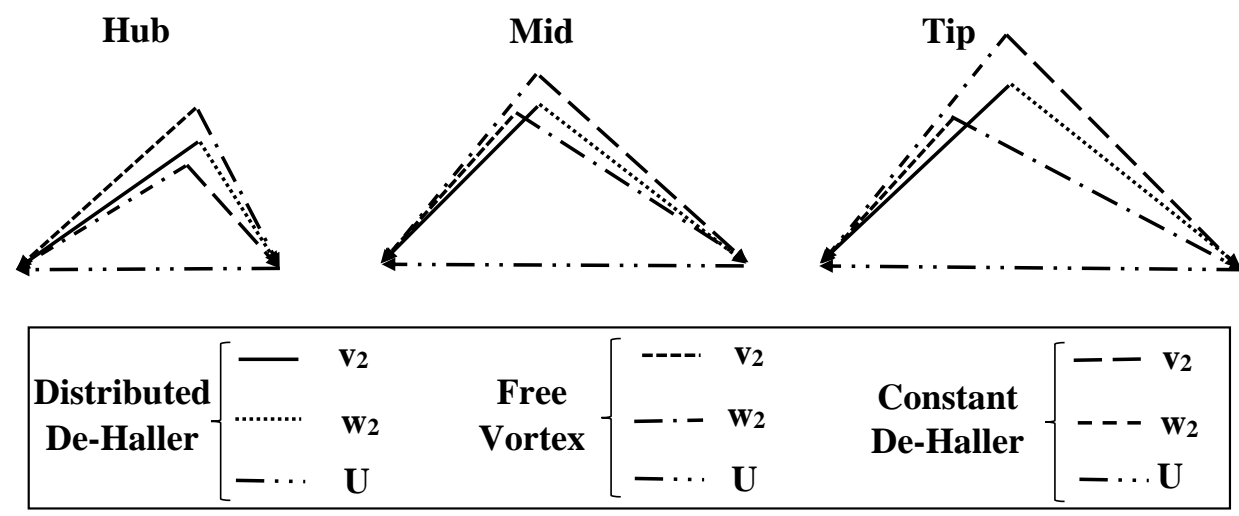

Fig. 1. Comparison of velocity triangles of the rotor outlet for three different design methods.

The switch function is a weighting function that implements a smooth span-wise de Haller distribution:

$$
\text { A. } r^{2}+B . r+C
$$

This function varies from 0.72 at $20 \%$ of the span to the de Haller number of a free vortex blade in the tip section with a diffusion factor less than 0.4 (Cumpsty 1989). A variable de Haller number distribution improves subsonic flow in the hub section and prevents flow separation in the tip section.

In addition to the point values for the span-wise distribution of the de Haller number, the derivative of Eq. (4) should be zero at $20 \%$ of the span to support a smooth variation of the de Haller number in Eq. (3). The coefficients in Eq. (4) are all calculated by implementing three boundary values. The distribution of the swirl velocity component can be obtained as follows by substituting Eq. (4) and Eq. (3) into Eq. (1) (the radial equilibrium equation) and solving the differential equation.

$v_{\theta 2}=\sqrt{(r \cdot \Omega)^{2} \cdot\left(1-d H^{2}\right)-d H \cdot \frac{\partial}{\partial r}(d H) \cdot\left[r \cdot v_{z 1}{ }^{2}+r^{3} \cdot \Omega^{2}\right]}$

The first term on the right-hand side of Eq. (5) represents a force vortex design. Unlike a free vortex design, the radial variation of enthalpy in Eq. (1) is not zero, which leads to non-free vortex variation of swirl velocity. Due to the radial variation of the de Haller number, the second term on the right-hand side of Eq. (5), which has a negative value, prevents a full force-vortex design.

The axial velocity component at the rotor outlet can be obtained by using a velocity triangle. Figure 1 shows the velocity triangles obtained with a constant de Haller number, the free vortex method, and a variable de Haller number. It is clear that a variably distributed de Haller number design generates a velocity triangle that is the average of the free vortex and constant de Haller number.

To evaluate the new design procedure, a new rotor was designed using the geometric and operating conditions of NASA rotor 67. The design conditions of NASA rotor 67 , which is presented in
Table 1, was chosen for the new rotor.

Table 1 Geometric parameters of DCA and MCA blade (Seyler \& Smith Jr, 1967).

\begin{tabular}{|c|c|}
\hline Parameter & Value \\
\hline Mass flow rate $(\mathrm{kg} / \mathrm{s})$ & 33.25 \\
\hline Rotational speed $(\mathrm{rpm})$ & 16043 \\
\hline Number of rotor blades & 22 \\
\hline $\begin{array}{c}\text { Inlet tip relative Mach } \\
\text { number }\end{array}$ & 1.38 \\
\hline Tip clearance $(\mathrm{mm})$ & 1 \\
\hline Isentropic efficiency & $90 \%$ \\
\hline Aspect ratio & 1.56 \\
\hline Inlet hub to tip ratio & 0.375 \\
\hline Outlet hub to tip ratio & 0.478 \\
\hline Inlet tip diameter $(\mathrm{cm})$ & 25.7 \\
\hline
\end{tabular}

Figure 2 shows the design point conditions including both geometrical parameters and operating conditions to be used in the design code to initialize the new rotor geometry. Despite ignoring the tip clearance in the design code, it was considered in CFD simulation. The code was developed based on the span-wise distributed de Haller number to extract the geometry of a new fan rotor. The most important inputs for the code were the mass flow rate, aspect ratio, hub-to-tip ratio, tip diameter, angular velocity of rotor, total pressure, and temperature, which were all the same as used for NASA Rotor 67. As shown in Fig. 2, the design code can evaluate other turbomachinery parameters, such as the reaction, loading, and diffusion factor at each blade section.

Blade counts were extracted through the optimum solidity, which was used for axial rotor design (Kerrebrock 1981). Standard double and multiple circular arc profiles were used to design airfoils in different span-wise sections of the blade (Seyler \& Smith Jr 1967). The design code gradually switches from a double circular arc (DCA) airfoil to a multiple circular arc (MCA) when the relative Mach number increases. The blade profile's 


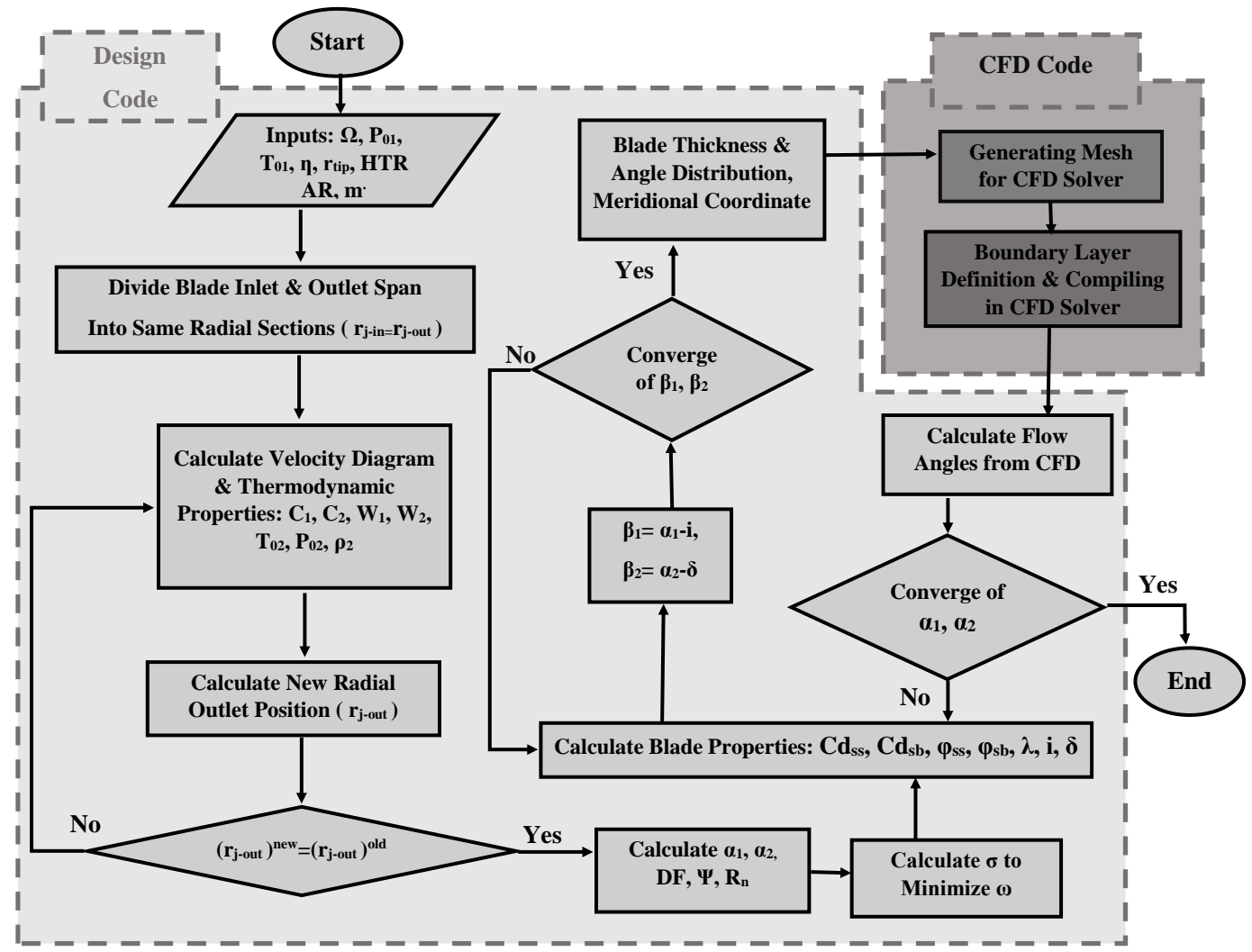

Fig. 2. Design flowchart.

Table 2 Geometric parameters of DCA and MCA blade (Seyler \& Smith Jr, 1967).

\begin{tabular}{|c|c|c|c|c|}
\hline $\mathrm{t} / \mathrm{Cd}$ & HTR & AR & re/t & $\varphi_{\text {ss }} / \varphi_{\text {sb }}$ \\
\hline \multirow{2}{*}{0.05} & $\begin{array}{l}\text { Inlet }=0.375 \\
\text { Outlet }=0.46\end{array}$ & 1.56 & 0.12 & $\begin{array}{c}0.5(0 \%<\operatorname{span}<50 \%) \\
\end{array}$ \\
\hline
\end{tabular}

characteristics are summarized in Table 2. Due to the different design methods, the incidence and the deviation angle were modified using CFD results. Thus, the design code was coupled with a 3D compressible viscous flow solver to obtain a modified geometry. Figure 2 shows the design procedure.

\section{NEW ROTOR DESIGN}

NASA rotor 67 was designed by the free vortex method and was used in a case study for comparison with a new designed fan. This study used a CFD flow solver only to modify the incidence and deviation angles. No optimization was used for airfoils in different blade sections, and only standard DCA and MCA profiles were used.

\subsection{Geometry of the Designed Fan}

Figure 3 compares the span-wise distribution of the blade angle for the new designed rotor with that of NASA rotor 67 . In contrast to the trailing edge, the blade angle at the leading edge is not affected by the design method. As seen, the new rotor has a lower camber angle in the hub section. The blade loading of the new designed rotor obviously increases at $30 \%$ of the span due to the higher blade camber angle. It seems that near the tip section, the blade angle distribution of the new rotor is similar to that of NASA rotor 67 and only shifts to lower values.

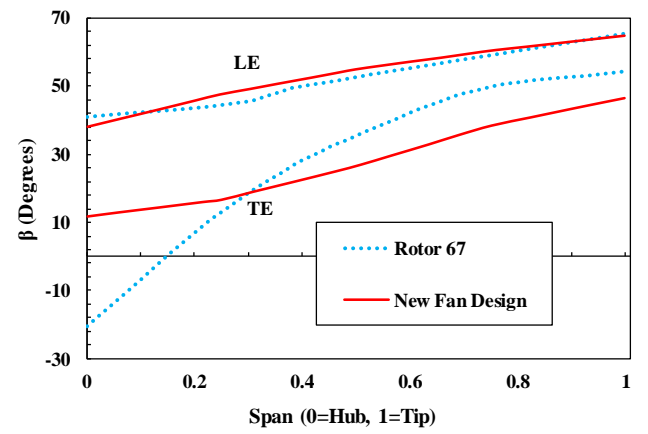

Fig. 3. Comparison of span-wise variation of blade angle of new fan design and NASA Rotor 67 at leading and trailing edges.

Figure 4 shows the blade sections in different spanwise sections. The camber clearly reduces in the hub section of the new fan design. As shown in Fig. 4 , the hub section of the new designed rotor is 


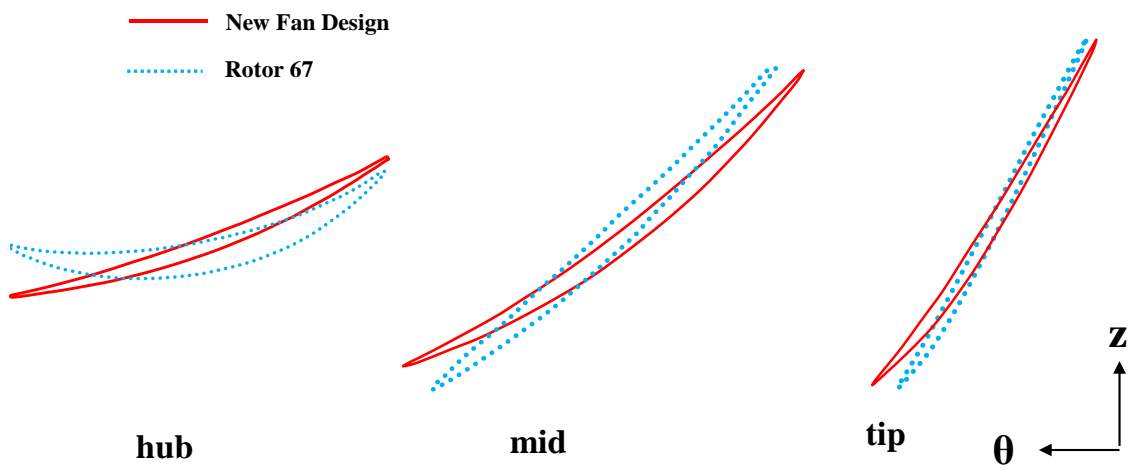

Fig. 4. Comparison of blade sections of the new fan design and NASA Rotor 67.

narrower than NASA rotor 67. A narrow hub section can be critical because of centrifugal stress in the root of the new blade. Indeed, some additional geometry modifications for more structural strength should be carried out in the future works. Figure 4 also shows the new rotor to have a higher chord length due to the higher loading in the middle and tip sections. The twist angle of the new fan design is much lower than that of NASA rotor 67 . The stacking method of the new designed rotor is the same as used in the Rotor 67. In other words, all center of area for all blade sections are stacked along the radial direction or $\mathrm{r}$-coordinate.

Figure 5 obviously shows the span-wise variations of the axial chord length in the meridional section. As seen, the shroud line is pushed down in the new designed rotor. Because the new design algorithm increases the blade loading especially through the tip section, the contraction ratio of the new meridional plane increases, which automatically pushes down the shroud line. Sloping the casing flow path can weaken or even remove the low energy fluids in the tip region, which is beneficial for the performance improvement of the new designed rotor. Despite all the differences in Fig. 5, the hub-to-tip ratio at the outlet of NASA rotor 67 is not affected by the design method.

\subsection{Validation of CFD results}

To evaluate the performance of the new rotor, a flow solver was used to extract a map of the new rotor. Experimental results for the NASA rotor 67 were used for validation (Seyler \& Smith Jr 1967). Figure 6 shows the flow domain to be divided into three main zones. Two stationary zones are defined at the rotor outlet and inlet, which help to implement the boundary conditions reported for NASA rotor 67 (Seyler \& Smith Jr 1967). As shown in Fig. 6, the flow direction, total pressure, and temperature are implemented at the inlet, and the average static pressure is implemented at the rotor outlet.

The blades, hub, and shroud were considered as noslip adiabatic walls. Because of the steady-state conditions, periodicity was beneficial for the computation cost of the rotor. As shown in Eqs. (6)(8), the relative velocity is substituted into the conservative equations of mass, momentum, and energy for the rotating domain. Coriolis and centrifugal accelerations were added to the convection term of Eq. (7) to model the rotation effects. A mixing plane was implemented as an interface to transmit values between the rotating and stationary zones (Okui et al. 2013).

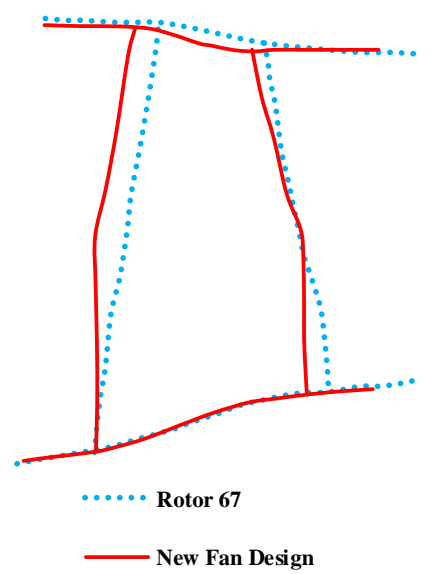

Fig. 5. Comparison of meridional geometry of the new fan design with NASA Rotor 67.

$\nabla \cdot \rho \vec{w}=0$

$\nabla . \rho \vec{w} \vec{w}+2 \rho(\vec{\Omega} \times \vec{w})+$

$\rho(\vec{\Omega} \times \vec{\Omega} \times \vec{r})=-\nabla p+\mu \nabla \bar{\tau}$

Shear stress transport was used as a turbulence model to capture the flow separation originating from shock waves and boundary layer interactions (Eisfeld et al. 2016). The viscous dissipation rate was considered as shown in Eq. (8).

$\nabla \cdot\left(\rho \vec{w} h_{0}\right)=-\nabla \cdot(k \nabla T+\bar{\tau} \cdot \vec{w})$ 


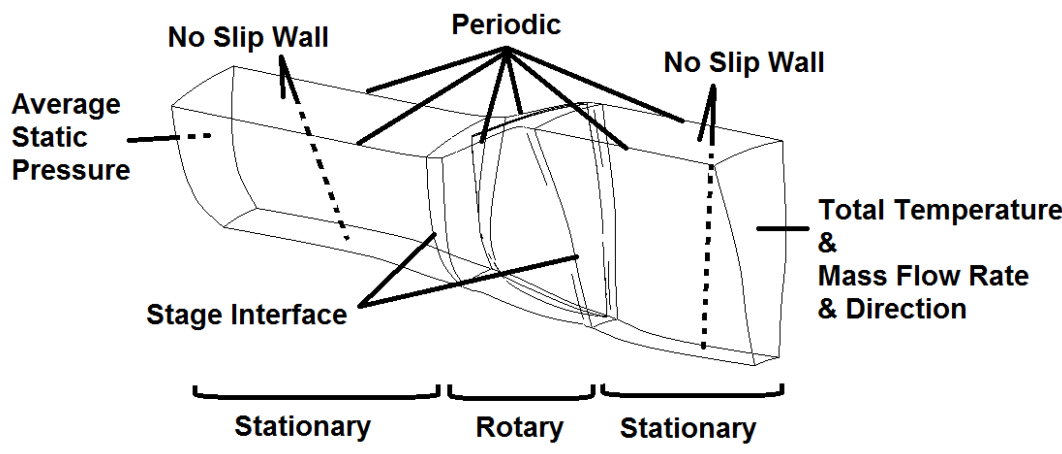

Fig. 6. Three zones and boundary conditions implemented in the fluid domain for the simulation.

Table 3 Four grid levels generated to investigate the effect of grid size on the performance

\begin{tabular}{|c|c|c|c|c|}
\hline Grid resolution & X-Coarse (XC) & Coarse (C) & Medium (M) & Fine (F) \\
\hline Number of grid level & $\mathrm{k}=1$ & $\mathrm{k}=2$ & $\mathrm{k}=3$ & $\mathrm{k}=4$ \\
\hline Number of elements & 176168 & 325796 & 542856 & 873892 \\
\hline Relative grid size & 1.705468 & 1.389432 & 1.171991 & 1 \\
\hline $\mathrm{r}_{\mathrm{p}}$ & 1.5545 & 1.56244 & 1.5716 & 1.5731 \\
\hline$\eta$ & 0.896077 & 0.900505 & 0.905765 & 0.9065 \\
\hline
\end{tabular}

A grid study was conducted to ensure that the CFD results were not affected by the grid size. As shown in Table 3, four grid levels are generated to investigate the effect of the grid resolution on the performance of the rotor. Eq. (9) and Eq. (10) represent the total pressure ratio and isentropic efficiency of the rotor, respectively, which were considered as two main non-dimensional parameters for the grid dependency of the results.

$r_{p}=P_{02} / P_{01}$

$\eta=\left(\left(P_{02} / P_{01}\right)^{\frac{\gamma-1}{\gamma}}-1\right) /\left(T_{02} / T_{01}-1\right)$

Richardson extrapolation was used to predict the exact solution to estimate the error according to Eq. (11) for each grid resolution, as shown in Fig. 7. For each grid level, the relative grid size was calculated using Eq. (12).

$$
\varepsilon=\frac{f-f_{\text {exact }}}{f_{\text {exact }}}
$$

$l=\left(\frac{N_{\text {fine }}}{N_{k}}\right)^{1 / 3}$

The third grid level with a medium resolution seemed to be optimal for both the efficiency and pressure ratio prediction of the transonic rotor. With this resolution, the relative error was less than $\boldsymbol{O}$ (0.001), which was small enough to be ignored in predicting the performance map. Figure 8 shows the optimum computational grid, which was used to predict the fan performance.

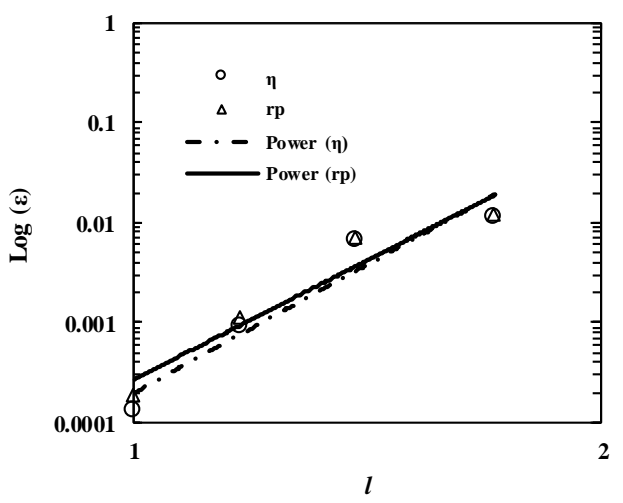

Fig. 7. Grid dependency in terms of error versus element size.

Figure 9 shows that the distribution of $\mathrm{y}^{+}$on the blade surface is less than 2 , which is small enough for k- $\omega$ SST model to capture near wall treatment without any wall function. Figure 10 compares the numerical and experimental performance map of NASA rotor 67, which shows only a small 

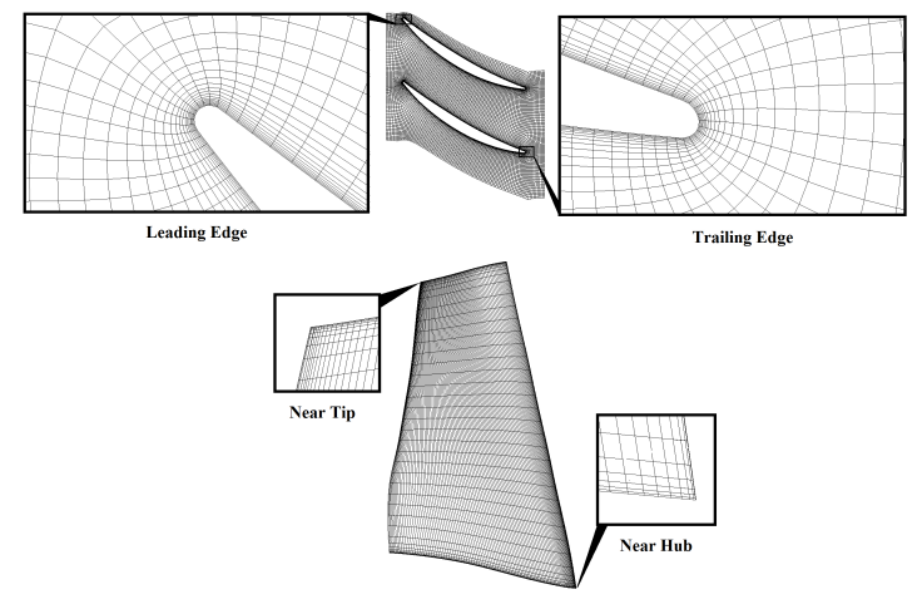

Fig. 8. Computational grid.

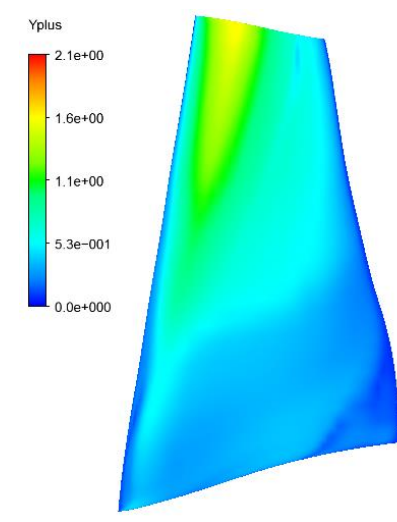

Fig. 9. Y+ distribution on blade surface. discrepancy between them. The discrepancies indicate that CFD under-predict the both the pressure ratio and isentropic efficiency.

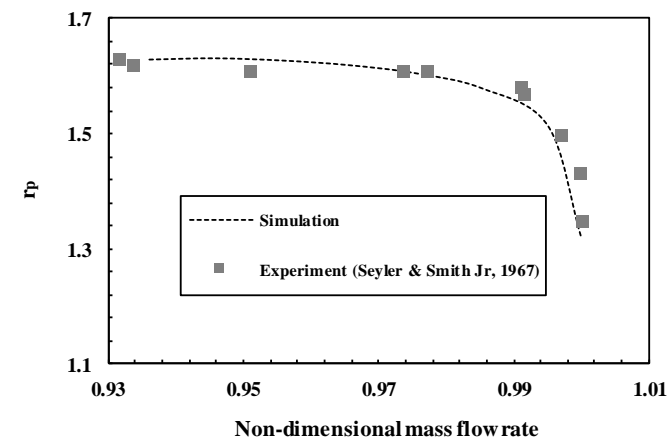

(a)

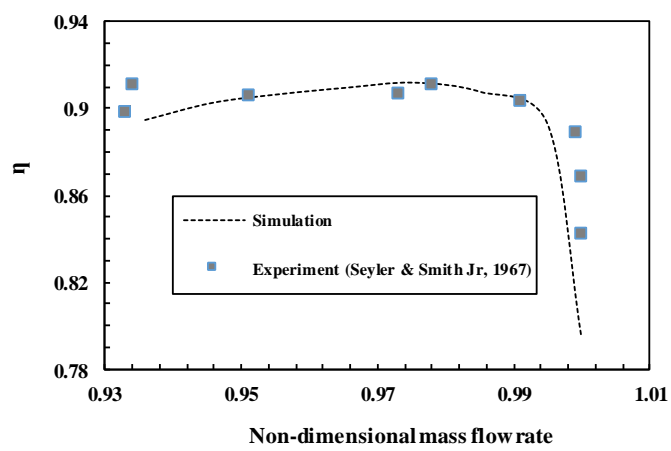

(b)

Fig. 10. Validation of CFD solver by experimental results of NASA rotor 67 ; (a) Pressure ratio; (b) Efficiency.

\section{RESULTS AND DISCUSSION}

\subsection{Comparison of New rotor and Rotor 67}

Figure 11 compares the blade loading at the design point of the new rotor with that of NASA rotor 67 for different span sections.

The blade loading is shown as the pressure distribution around the blade at each radial section. According to Fig. 11, despite a lower blade camber angle in the hub section, the pressure difference between pressure and suction sides of the blade increases. This means that the losses sometimes can overcome the generated pressure ratio. There is obviously higher blade loading in the new rotor at the mid- and tip-section, which is mostly due to the modified pressure distribution, especially after the shock wave. The enhanced loading distribution of the new designed fan was mostly due to the efficient geometry, including the camber and thickness distribution. Achieving such a geometry was quite dependent on the modified span-wise loading distribution.

The Mach number distribution at the design point of the new designed fan is compared with that of NASA rotor 67 in Fig. 12. There is a smaller wake in the hub section of the new designed fan, which is mostly due to the lower blade camber. In addition to efficiency, the modified flow in the hub section can improve the pressure recovery. It should be considered that the local relative Mach number on the suction side of the new designed fan is higher than that of NASA rotor 67. Figure 11 (c) showed that the higher Mach number before the shock wave leads to a stronger shock with more pressure rise on the suction side. Figure 12 also shows that both the first and second shock waves are much stronger in the new fan, which can generate a higher pressure ratio. The shock waves are also generated farther from the leading edge, which is beneficial for increasing shock stability, especially in off-design conditions.

Figure 13 presents the performance map of the new fan at both design and off-design conditions, and compares to that of NASA rotor 67. As seen, the pressure ratio and isentropic efficiency of the new fan are enhanced by about $3 \%$ and $1.5 \%$, respectively. Enhanced performance is also 
observed at off-design rotor speed. Another important characteristic of the new fan is the shifted surge inception point, which can be beneficial for the maneuverability of the aircraft. The point of surge inception was assumed to be at maximum pressure ratio for every rotational speed of rotor. Consequently, the difference between the mass flow at operating and surge inception point was used in Eq. (13) to define the surge margin. Table 4 compares the surge margin of NASA rotor 67 with that of the new rotor for different rotor speeds.

$$
S M=\left(\dot{m}_{\text {operating }}-\dot{m}_{\text {surge }}\right) / \dot{m}_{\text {surge }} \times 100
$$

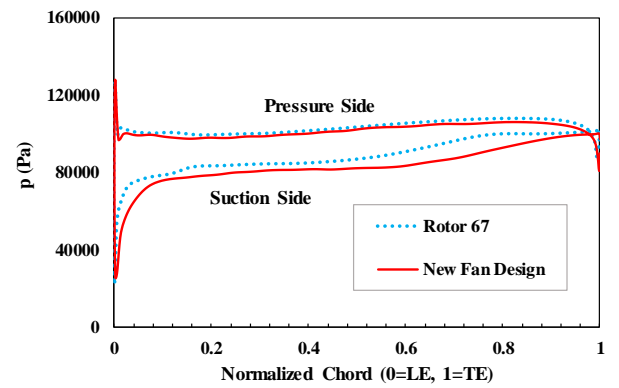

(a) $\mathrm{Hub}$

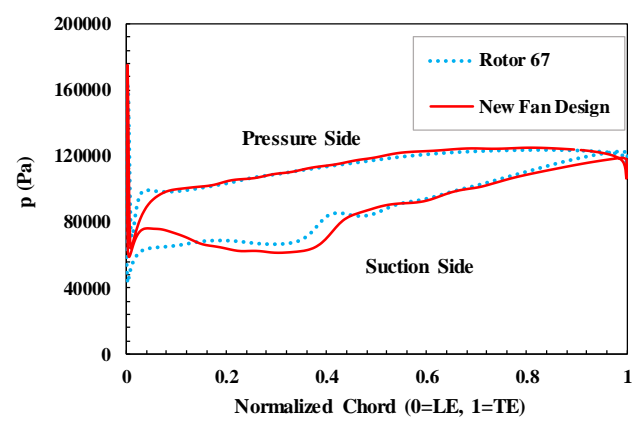

(b) $\quad$ Mid

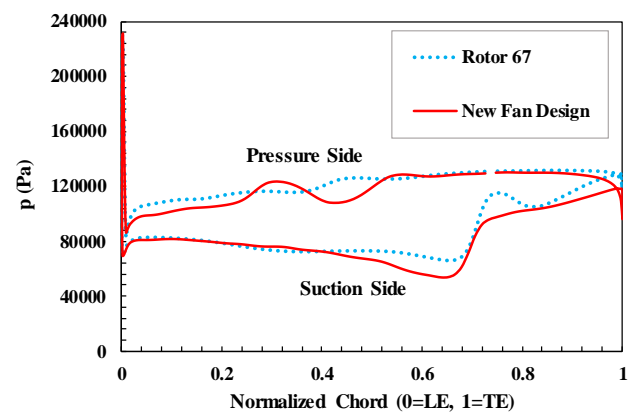

(c) Tip

Fig. 11. Loading represented by pressure distribution around different blade sections.

Figure 14 compares the streamlines near the hub for NASA rotor 67 and the new designed rotor. As seen, the streamlines of the NASA rotor 67 tend to migrate along the radial direction more intensively than the new designed rotor. This decreases the outlet flow momentum and efficiency near the hub of the NASA rotor 67 compared to the new designed rotor.

Table 4 Surge margin of rotors.

\begin{tabular}{|c|c|c|}
\hline \multirow{2}{*}{$\% \Omega_{\mathrm{N}}$} & \multicolumn{2}{|c|}{ SM } \\
\cline { 2 - 3 } & Rotor 67 & New Rotor \\
\hline 80 & 10.71429 & 14.28571 \\
\hline 90 & 6.666667 & 10 \\
\hline 100 & 4.041325 & 9.022556 \\
\hline 110 & 0.724638 & 5.249463 \\
\hline
\end{tabular}

As mentioned, a shock wave near the blade's trailing edge is more stable and helps to enhance the surge margin. It seems that an even more efficient fan can be designed by optimization of the blade airfoil or stacking line of the rotor.

Figure 15 indicates that NASA rotor 67 has a lower outlet momentum near the hub than the new designed rotor. Figure 16 shows the span-wise distribution of efficiency for NASA rotor 67 and the new designed rotor at different rotational speeds, which obviously indicates the new rotor to have a higher efficiency in most of the blade span-wise sections. The enhanced efficiency of the new rotor mostly originates from the implemented blade loading. The enhanced efficiency of the new designed rotor near hub compared to NASA rotor 67 (Fig. 16) is also because of the less tendency of the hub streamlines to migrate along the radial direction (Fig. 14).

Figure 17 compares the meridional pressure distribution of the new rotor with that of NASA rotor 67. As shown, the outlet pressure for both the new rotor and NASA rotor 67 increases from hub to tip due to the radial equilibrium hypothesis in their preliminary designs. Figure 18 compares the velocity streamlines on the meridional planes of the new rotor and NASA rotor 67 . As seen, there is no distortion for the streamlines, which indicates the radial equilibrium is satisfied in the new rotor design.

Although the current study focused on a rotor design method, some flow characteristics including flow angle, axial and circumferential velocity at the rotor outlet were numerically obtained for a rough estimation of the stator design. Figure 19, 20 and 21 respectively compare flow angle, axial and circumferential velocity at the outlet of the new rotor and NASA rotor 67. Despite using a different design method for rotor, flow angle has not been changed significantly in the tip region of the new rotor outlet compared to NASA rotor 67 (Fig. 19). In addition, the stator can be designed without any risk of flow separation near the hub due to a lower flow angle in the hub section of the new rotor. Figure 20 shows that the axial velocity at the outlet of the new rotor near the tip section is about $15 \%$ higher than that of NASA rotor 67 . Indeed, the higher axial velocity results in more challenges for designing the stator because of a higher Mach number on the suction side of the stator blade, which can be resolved by an aerodynamic optimization in the future works. Figure 21 


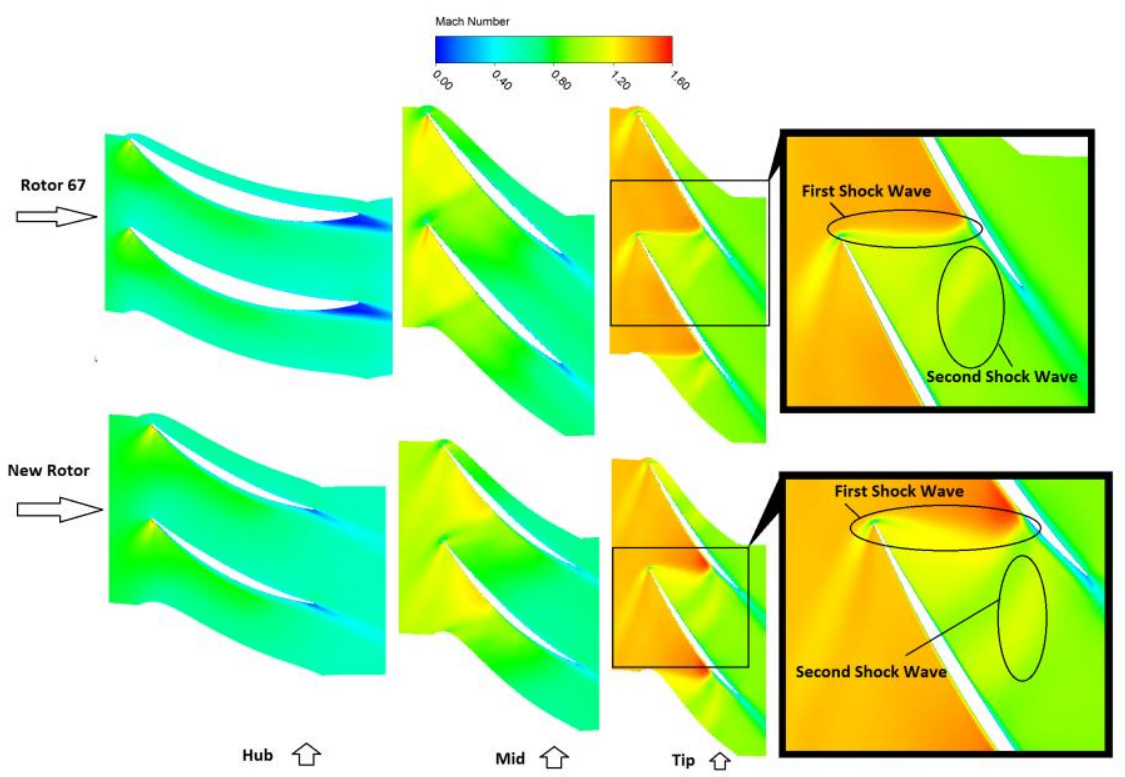

Fig. 12. Relative Mach number distribution around the new fan design and NASA Rotor 67.

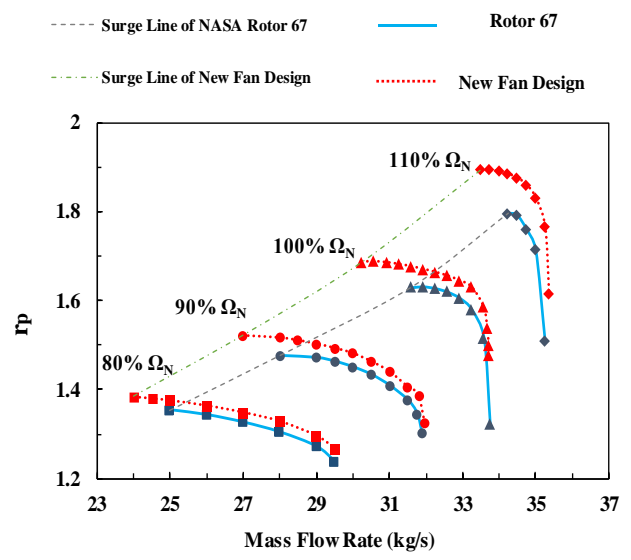

(a)

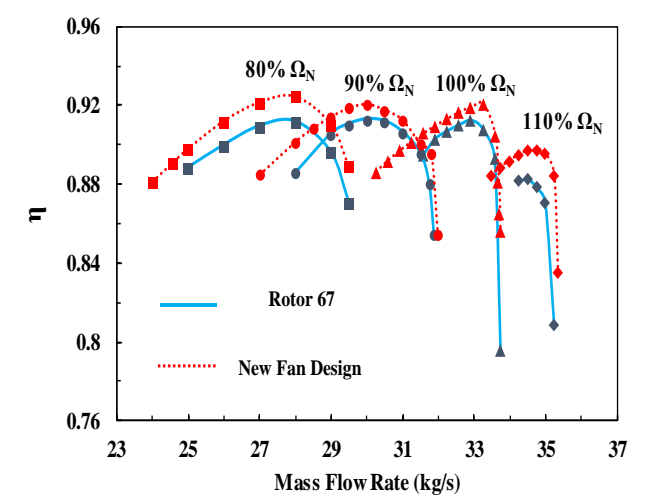

(b)

Fig. 13. Comparison of performance map of the new fan design with Rotor 67; (a) Pressure ratio; (b) Efficiency.
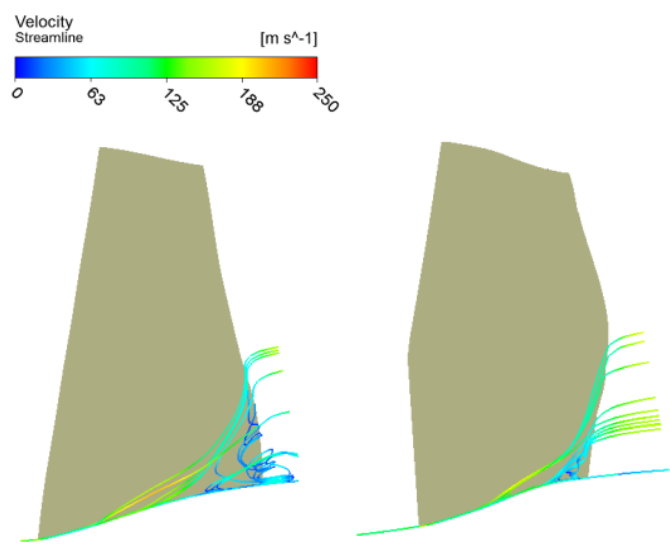

Fig. 14. Comparing 3D streamlines near hub section.

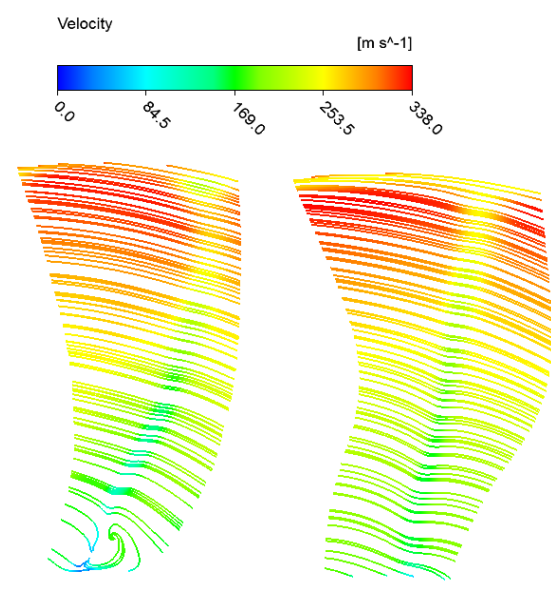

Rotor 67

Fig. 15. Comparing streamlines in pitch-wise. 


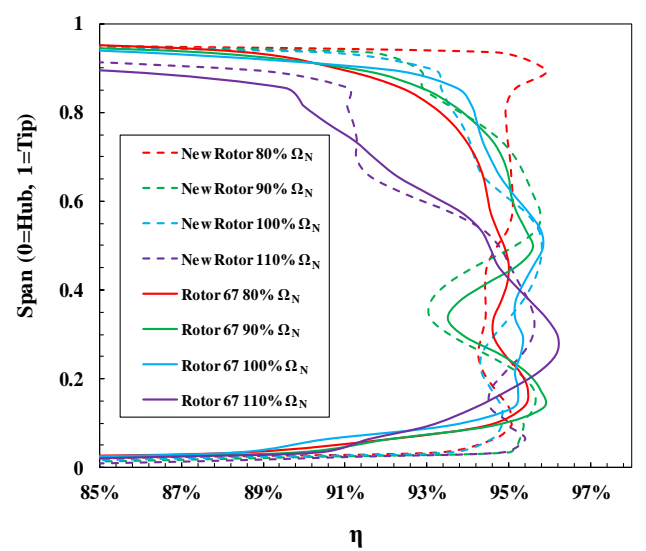

Fig. 16. Comparing efficiency span-wise distribution.

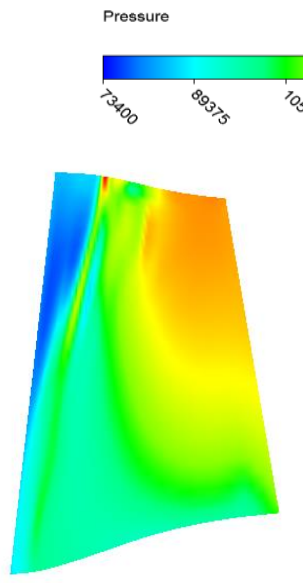

Rotor 67
Fig. 17. Static pressure distribution in meridional section.

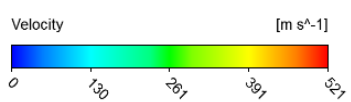

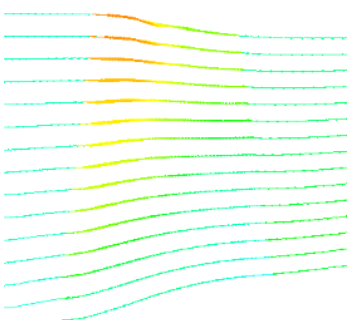

Rotor 67

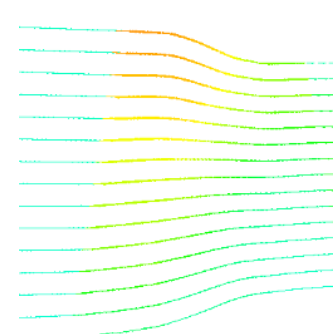

Fig. 18. Radial equilibrium is satisfied in rotor design.

compares the span-wise distribution of circumferential velocity for NASA rotor 67 and the new rotor. As shown, there is a higher circumferential velocity in mid and tip of the new rotor, which represents a higher loading factor for the new rotor compared to NASA rotor 67.

Figure 22 compares the diffusion factor for the new rotor and NASA rotor 67 . As shown, the new rotor

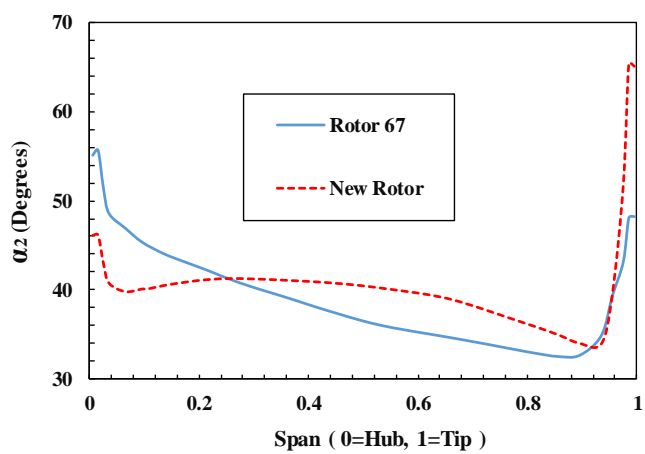

Fig. 19. Span-wise distribution of flow angle in rotor outlet.

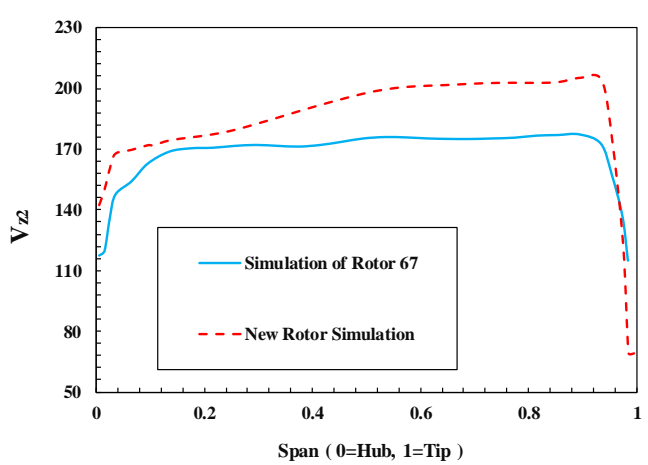

Fig. 20. Span-wise distribution of axial velocity in rotor outlet.

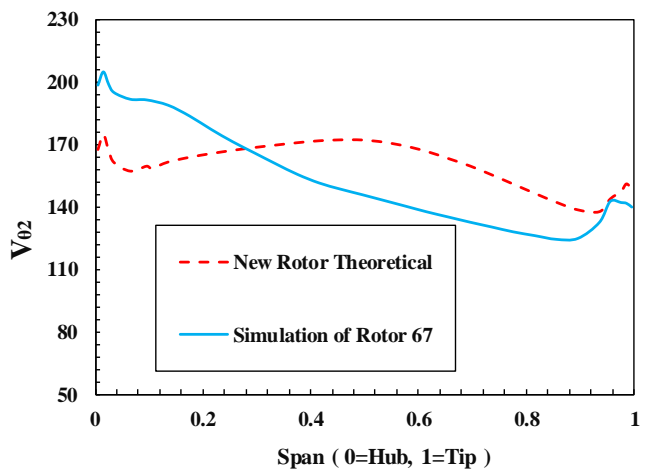

Fig. 21. Span-wise distribution of axial velocity in rotor outlet.

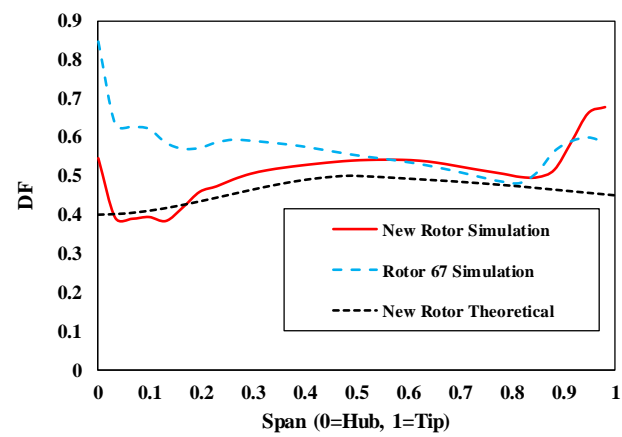

Fig. 22. Span-wise distribution of diffusion factor. 


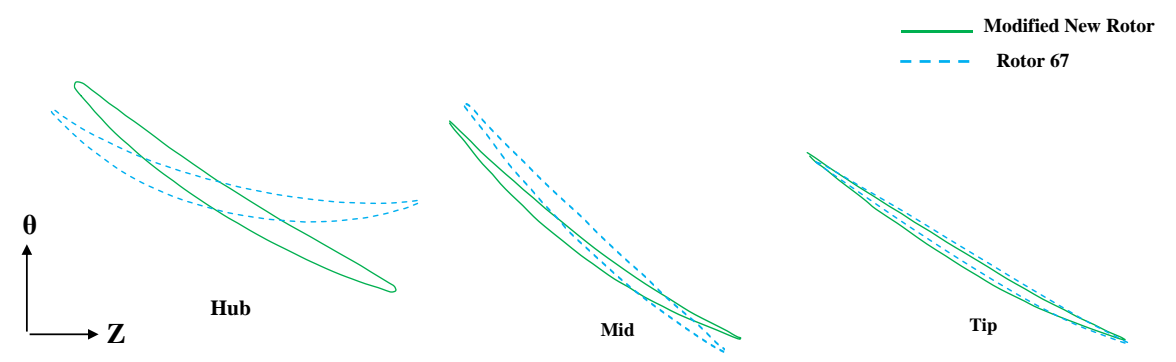

Fig. 23. Comparison of the blade sections for the modified new rotor and NASA Rotor 67.

has a lower diffusion factor near the hub section that gradually tends to the diffusion factor of NASA rotor 67 near the tip section. Figure 22 also compares the diffusion factors obtained by the preliminary design and CFD simulation of the new rotor, which shows a good agreement except near the hub and tip.

\subsection{Effect of Blade Thickness}

As mentioned in section 2, the thickness to chord ratio of the new designed rotor blade was considered to be constant while this is not constant for NASA rotor 67. In order to have more identical design to NASA rotor 67 , the thickness to chord ratio of the new rotor was modified based on the thickness distribution of NASA rotor 67. Figure 23 compares the blade sections for NASA rotor 67 and the new rotor with the modified thickness. Figure 24 compares the performance maps of the new rotor, new rotor after the thickness modification, and rotor 67 at nominal rotor speed. As it is obvious, the pressure ratio is not effected by the blade thickness. The maximum efficiency near the design point is reduced, and the surge margin is slightly restricted after the thickness modification. However, efficiency, pressure ratio and surge margin are still higher than that of NASA rotor 67. Indeed, the efficiency loss near the design point can be resolved by optimizing the blade profile in the future works.

\section{CONCLUSION}

A new fan was designed using a distributed spanwise de Haller number, which resulted in 3\% higher pressure ratio in comparison to NASA Rotor 67. The results showed that the higher pressure ratio mostly originated from the higher camber angle, modified blade loading, and stronger shock waves. The stronger shock waves were suitably controlled by the blade loading implemented in the design procedure to maintain the efficiency. Besides the higher pressure ratio, the enhanced efficiency of the new fan design was mostly due to the lower losses. Additionally, shock wave stability made the surge margin be larger by 1.5 times. All of these advantages were related to the efficient characteristics of the geometry, such as the camber and thickness distribution of the blades. The efficient geometry originated from the modified span-wise loading distribution used in the design procedure. No optimization was used to modify the blade profiles at different blade sections. It seems that besides CFD flow solvers, preliminary design methods for turbomachinery have the potential to obtain more optimal design methods.

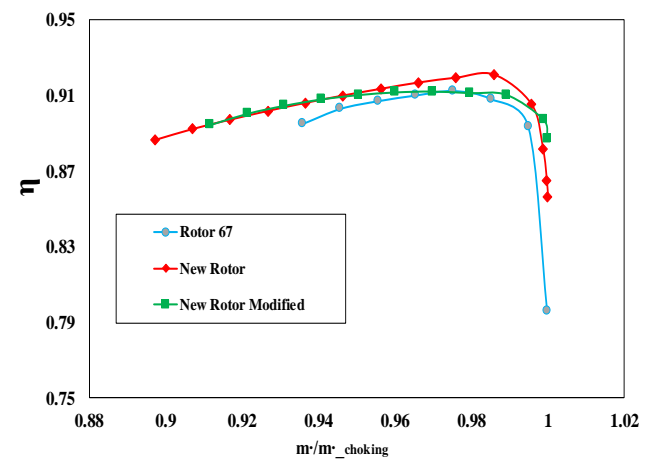

(a) Efficiency

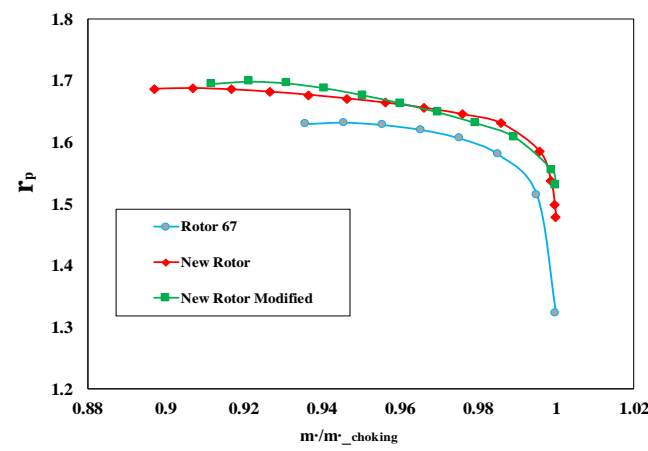

(b) Pressure ratio

Fig. 24. Comparison of Performance maps for the new rotor, the modified new rotor and NASA rotor 67.

\section{ACKNOWLEDGEMENTS}

This work was supported by the Brain Pool Program through the National Research Foundation of Korea (NRF-2019H1D3A2A01061428), which is funded by Korean government (MSIT). Partial support was also obtained from the National Research Foundation of Korea (NRF) grant, which is funded by the Korean government (MSIT) (No. 2020R1A5A8018822, No. 2018R1A2B2007117). 


\section{REFERENCES}

Benzakein, M. J. (2014). What does the future bring? A look at technologies for commercial aircraft in the years 2035-2050. Propulsion and Power Research 3(4), 165-174.

Choi, S. M., J. S. Lee, R. S. Myong, H. N. Jo and J. W. Kim (2020). Schlieren visualization of micro gas turbine exhaust plume with different shapes of nozzle. Journal of Visualization 23, 565-576.

Cumpsty, N. A. (1989). Compressor aerodynamics. Longman Scientific \& Technical.

Danish, S. N., S. R. Qureshi, M. M. Imran, S. U. D. Khan, M. M. Sarfraz, A. El-Leathy, H. AlAnsary and M. Weie (2016). Effect of tip clearance and rotor-stator axial gap on the efficiency of a multistage compressor. Applied Thermal Engineering 99, 988-995.

Dickens, T. and I. Day (2011). The design of highly loaded axial compressors. Journal of Turbomachinery 133(3), 31007

Eisfeld, B., C. Rumsey and V. Togiti (2016). Verification and validation of a secondmoment-closure model. AIAA Journal, 15241541.

Gao, K., Y. Xie and D. Zhang (2017). Effects of stator blade camber and surface viscosity on unsteady flow in axial turbine. Applied Thermal Engineering 118, 748-764.

Kerrebrock, J. L. (1981). Flow in transonic compressors. AIAA Journal 19(1), 4-19.

Luo, J., C. Zhou and F. Liu (2014). Multipoint design optimization of a transonic compressor blade by using an adjoint method. Journal of Turbomachinery 136(5), 51005 .

Okui, H., T. Verstraete, R. A. Van den Braembussche and Z. Alsalihi (2013). Threedimensional design and optimization of a transonic rotor in axial flow compressors. Journal of Turbomachinery 135(3), 31009.

Oyama, A., Liou, M. S. and S. Obayashi (2004). Transonic axial-flow blade optimization: Evolutionary algorithms/three-dimensional Navier-Stokes solver. Journal of Propulsion and Power 20(4), 612-619.

Ren, X. and C. Gu (2016). A numerical study on the tip clearance in an axial transonic compressor rotor. Applied Thermal Engineering 103,
$282-290$

Samad, A., K. Y. Kim, T. Goel, R. T. Haftka and W. Shyy (2008). Multiple surrogate modeling for axial compressor blade shape optimization. Journal of Propulsion and Power 24(2), 301-310.

Saravanamuttoo, H. I. H., G. F. C. Rogers and H. Cohen (2001). Gas turbine theory. Pearson Education.

Seyler, D. R. and L. H. Smith Jr (1967). Single stage experimental evaluation of high Mach number compressor rotor blading. Part IDesign of rotor blading.

Shahsavari, A. and M. Nili-Ahmadabad (2016). Aerodynamic design of a highly loaded axial flow fan rotor using a novel one-dimensional design method with its numerical simulation. In Proceedings of the ASME Turbo Expo (Vol. 2A-2016)

Shahsavari, A. and M. Nili-Ahmadabadi (2017). Investigation of an innovative non-free vortex aerodynamic procedure to design a single-stage transonic compressor. Proceedings of the Institution of Mechanical Engineers, Part G: Journal of Aerospace Engineering

Sun, S., S. Wang, S. Chen, C. Tao, L. Cai and J. Chen, (2019). The impact of various forward sweep angles on the performance of an ultrahigh-load low-reaction transonic compressor rotor. Applied Thermal Engineering 150(August 2018), 953-966.

Tang, X., J. Luo and F. Liu (2017). Aerodynamic shape optimization of a transonic fan by an adjoint-response surface method. Aerospace Science and Technology 68, 26-36.

Walther, B. and S. Nadarajah (2013). Constrained adjoint-based aerodynamic shape optimization of a single-stage transonic compressor. Journal of Turbomachinery 135(2), 21017.

Wu, C. H. and L. Wolfenstein (1950). Application of radial-equilibrium condition to axial-flow compressor and turbine design. NACA Technical Report 955.

Yang, Y., A. Sciacchitano, L. L. M. Veldhuis and G. Eitelberg (2018). Analysis of propellerinduced ground vortices by particle image velocimetry. Journal of Visualization 21, 3955 . 